\title{
The DAM marketing communication creation process: A new way of working
}

\author{
Cathal McKee \\ is the founder and creative director of $\mathrm{CMK}$. He has 19 years of experience working in the areas of design, branding, \\ advertising and the internet. A graduate of Ravensbourne College of Art in London, he is now based in Amsterdam. \\ Cathal concentrates his work on looking at the impact of new technologies, such as digital asset management \\ systems, and rewriting creative processes to deliver even more integrated experiences. Advertising Everywhere is a \\ platform of future insights into the relationship between brands, advertising and the user.
}

Keywords: digital asset management (DAM), creative direction, content creation, information architecture, Advertising Everywhere, visual intellectual property (VIP), creative process

Abstract Digital asset management (DAM) is having an impact on content creation for all media forms, first and foremost traditional advertising and printed media. With the implementation of DAM systems in many of the Fortune 100 companies, the role between the creative agency and the corporate client is changing. To many agencies, DAM is viewed as a direct threat to their existing business model, as template creation automates many traditional DTP tasks. Next to the initial savings and cost efficiencies, the creative agency needs to change its role and the service it offers. The DAM marketing communication creation process is a way of working that provides clients and agencies with guidance and support in all aspects of the process, helping to create a better understanding of the impact within an organization and the relationship with its creative agencies.

Cathal McKee CMK

Herengracht 313 1016 AV Amsterdam The Netherlands.

Tel: +31614450003 Email:

cathal@cmkoffice.com

\section{INDUSTRY CHANGE}

Creating change in any industry needs an enormous effort in setting new targets, goals and visions. In the cerebral world of creative marketing words like concept, image, brand and communication, the idea of any system or application to "manage" creativity frightens the life out of most openminded creative agencies. Most critics complain that the control of the creative quality will be lost, putting creativity into the "deadly" hands of the client. The thought of clients "being creative" and making their own advertisements sounds like a recipe for disaster. Especially after so much carefully nurtured brand building, agencies see their core business value lost to cold hard efficient technology.

On the contrary, digital asset management (DAM) systems offer even more opportunity to create focused messaging, tighter brand control and most importantly, and something overlooked in every DAM presentation that I have seen, a DAM system brings the message to the consumer as quickly as possible. The potential of DAM systems to offer the flexibility to react to 
topical events and change the message as quickly as possible, so bringing any brand message into context within our daily lives, should be the end goal of any creative director or marketing manager,.

\section{THE NEW MINDSET}

The DAM marketing communication creation process needs to provide a new way of working with a new mindset to see the opportunities and help bring out the benefits. DAM creates the enabling conditions to manage and delegate activities in a more structured and efficient manner. It helps to place problems and issues in communication categories, as opposed to the traditional collision of media choice and creative thinking where time and energy is lost in multiple correction rounds and changes of opinion. Using this process helps discussions on brand placement, with media choice becoming more effective and reactive to market changes. Wouldn't it be great for any brand to react to any world or topical event within 24 hours?

\section{CONTENT AND ASSET MAPPING}

Every brand has a literal wealth of assets created over years and years of advertising and marketing communication (see Table 1). These assets include brand names, sub-brands, key visuals, copy, photography, illustrations, video imagery, such as commercials, and, in recent times, the interactive brand identity — the way a brand clicks, and reacts to its user. All these assets are mapped into a database. Traditionally, assets are mapped by their media formats and document types. Yet what should be interesting to the creative director is the opportunity to give a rank and value to each and every asset, so creating a hierarchical content map.

Content and asset mapping assigns a hierarchical value to assets. The layers

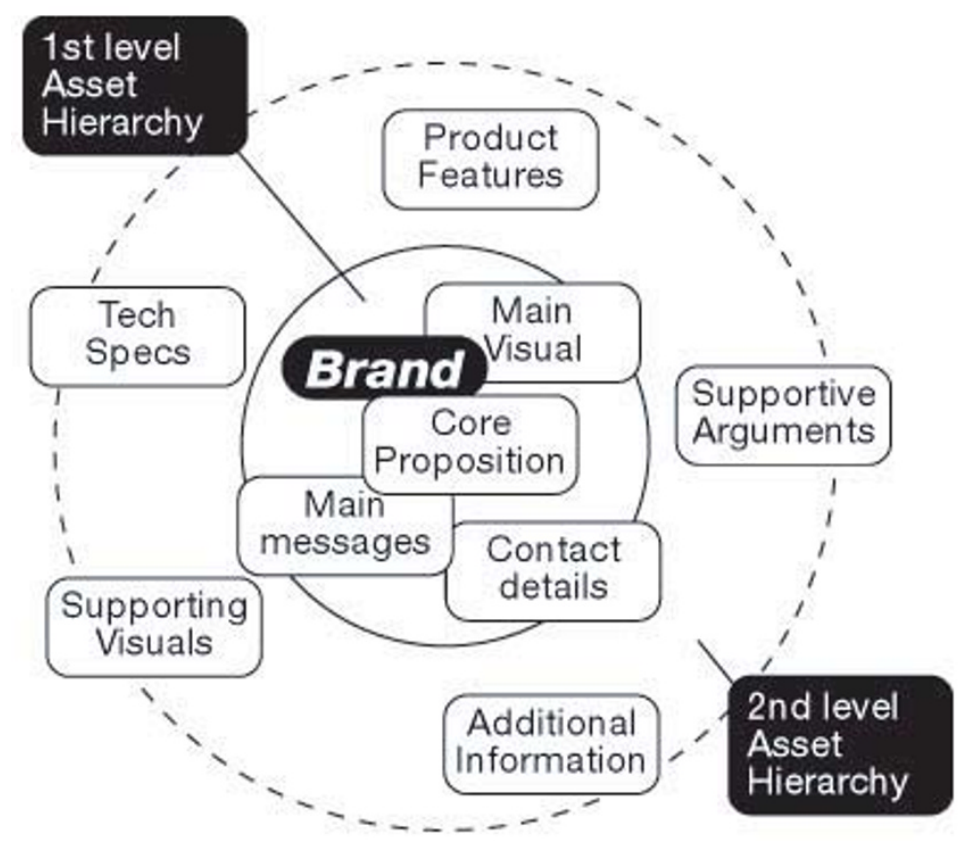

Figure 1: Content mapping of first and second hierarchy of information 
help to focus both client and agency to make hard decisions on what messaging has the most importance, establishing a hierarchy into which images, text and illustration will have the most impact. This kind of mapping helps to create clearer messages, communication with more impact, and prepare clients for creative better agency briefings.

\section{IDEA CREATION}

Every brand is busy trying to put ideas and propositions into peoples' minds. These ideas, if successful, build brands and are one of the most important assets a brand can have. Ideas are initially intangible, but once converted to data, the idea can be worked into texts or visuals. The development of the proposition is integral to the brand's core. Idea generation is the creative process whereby unique propositions are mapped, defined and created into words and images. The goal of this exercise is two-fold: first to find the unique and fresh message that makes the proposition unique in its market, and secondly to extract its uniqueness from the other products or services that the company offers, while still being an integral part of the whole offer. Once again, looking at asset creation from the application viewpoint is far different from traditionally looking at the idea generation concept creation as something that is presented in a media form. Formalizing idea creation into assets can start to make the creative team look more closely at the relationships between the assets. Does that picture have any relationship with that text? Does the text have the same tone of voice as the previous five advertisements? Is the brand style intact on this advertisement? These are the type of questions that are made possible by approaching the idea creation phase with asset management in mind.

\section{CREATING VISUAL INTELLECTUAL PROPERTY (VIP)}

Advertising images and texts form the basis of a brand's visual intellectual property (VIP). We call them the VIP brand assets. These assets, words, taglines, images and key visuals, are an integral part of the brand's visual identity. VIP for a brand has three elements:

- brand identity: the logo, typeface, colors;

- brand imagery: photography, illustrations, animations;

- brand messages: taglines, copy and textual elements, sub-branding, product naming.

Each brand asset is crafted with the utmost care and attention to detail. Any new element or asset to the identity needs to fit visually within the existing identity and be prepared for the formats in which it will be published. Each asset needs to be legally trademarked, so that no other company can claim ownership of it.

When the concept ideas have been approved and converted to data, the process begins in creating the brand's visual assets. The creation of VIP is the actual production of these assets.

Some of them may be mandatory and already developed, such as a logo; others may need to be created for campaign purposes or the optimization of the brand identity. Each and every asset created has value. Understanding the precious nature of each asset is fundamental to how they are used. Just throwing them into any size, form of 
advertisement or internet page will lead to brand dilution.

\section{INFORMATION ARCHITECTURE}

Every asset has its place, not only within the database, but also within the architecture of the information. The process of information architecture is to deliver the right messages and assets with the right media. Traditionally, the idea of analyzing the placement of each and every asset doesn't exist. Only the media choice is judged and the operation is performed by the media planner. This has led to the multiple recreation of assets with little regard to their relationships with other assets. With DAM systems it is now possible to deconstruct any piece of information and relate it back to the individual asset. In this context, the asset becomes the focus, thus bringing the attention back to, say, a couple of key elements that are repeated or adapted to the architecture of the medium. The skill of the information architect (see Table 1) is to concentrate on mapping communication and brand assets to the media. Based on the media plan, information architecture creates an overview of what assets are being placed where and in which formats.

The information architecture part of this process is not necessarily a part of the process that immediately follows the VIP process, but should be seen as one that complements the asset creation process. Often, due to target group or localization, there needs to be careful adjustment to the brand assets. Each medium is mapped; each brand message is developed, translated and resized to the format and medium available. What is interesting about this activity is that it helps the creative director to think about the number of assets that are already available or to be created. In any communication piece, the fewer the assets, the more impact the piece will have. So, the first priority for any DAM system is to minimize the number of assets available. Of course, this conflicts with one of the key selling points of any DAM system, ie that it can manage many assets.

The information (media) architect is key to the creative process. He/she is the person who manages which assets are being placed into which media. For each media item, the information architect can tell exactly which asset is placed where, so offering the client an exact overview not only of the media, but also the exact assets per media item. More controlled brand management does not exist in the market today. The management is based on traditional print-based media and template creation. In realizing that creating too many variables in the assets leads to more diffused or dysfunctional communication, the information architecture can help control unnecessary asset creation.

Table 1: The information architecture across campaigns showing assets used

\begin{tabular}{llllllc}
\hline Campaign & Brand name & Key visual & Headline & Copy & Pay off & $\begin{array}{c}\text { Secondary } \\
\text { visual }\end{array}$ \\
\hline 1 & $\mathrm{X}$ & $\mathrm{X}$ & $\mathrm{X}$ & $\mathrm{X}$ & $\mathrm{X}$ & \\
2 & $\mathrm{X}$ & $\mathrm{X}$ & $\mathrm{X}$ & & $\mathrm{X}$ & $\mathrm{X}$ \\
3 & $\mathrm{X}$ & & $\mathrm{X}$ & $\mathrm{X}$ & $\mathrm{X}$ & \\
\hline
\end{tabular}


ADVERTISING EVERYWHERE

The DAM system can manage assets and templating in traditional media forms.

Advertising Everywhere, however, goes beyond the traditional ways we consider advertising. In addition to automation of traditional media advertising, there is an explosion of growth in advertising in new and innovative places. While television, print and radio are still the main media choices for most advertising campaigns, there are many new and different ways that a communication piece can be received and customized by your customers. Categories such as ambient, buzz, digital, sms, urban and guerrilla are all new forms of marketing and communication. These new media forms need new ways to manage assets. To understand this new wave, think Lance Armstrong's Livestrong armband, or the guy who sold his forehead on
eBay for $\$ 27,000$, or the possibility of advertising on Google earth, the visual geographical search software. I have categorized all these new possibilities into the name Advertising Everywhere. It is happening parallel to the growth of DAM systems.

\section{CREATIVE DIRECTION}

With the growth and implementation of DAM systems into the advertising landscape, the role of the creative director and the creative agency is creating an enormous need for new processes and new thinking in finding the best practices and new opportunities in working with these systems. In a few years' time we will look back at how much we have changed our way of working and our focus on creating the best assets for the brand. 